\title{
A violência intrafamiliar e o adolescente que vive com HIV/AIDS por transmissão vertical: análise dos fatores de proteção e de vulnerabilidade
}

\author{
Domestic violence and the adolescent that was infected with HIV \\ through vertical transmission: analysis of protection \\ and vulnerability factors
}

Ana Cláudia Mamede Wiering de Barros ${ }^{1}$

Olga Maria Bastos ${ }^{1}$

Marcos Vinicius da Silva Pone ${ }^{1}$

Suely Ferreira Deslandes ${ }^{2}$

${ }^{1}$ Departamento de Pediatria, Instituto Fernandes Figueira Fundação Oswaldo Cruz. Av. Rui Barbosa 716, Flamengo. 22250-020 Rio de Janeiro

RJ.acmamede@iff.fiocruz.br

${ }^{2}$ Departamento de Ensino,

Instituto Fernandes

Figueira.

\begin{abstract}
The scope of this study was to analyze protection and vulnerability factors in physical and psychological domestic violence among adolescents infected with HIV/AIDS through vertical transmission. This group is especially susceptible as they have vulnerability factors such as chronic disease, orphanhood with consequent change of caregivers and impaired body image. The research was conducted in a public hospital. The first stage used the Parent Child Conflict Tactics and the Degree of Psychological Violence Scale to determine what domestic violence occurred. In the second qualitative stage, the adolescents who scored the most or the least for violence in the quantitative instrument were interviewed. The questionnaires and the interviews revealed a high prevalence of physical and psychological violence and abusive domestic dynamics, thereby corroborating the extant literature. In view of the consequences of this kind of violence, it is essential that the health professional should be able to identify violent situations and recognize vulnerability factors and to promote protection factors against ill-treatment.
\end{abstract}

Key words Domestic violence, HIV/AIDS, Vulnerability factors, Protection factors
Resumo O objetivo deste trabalho foi o de analisar os fatores de proteção e de vulnerabilidade à violência intrafamiliar física elou psicológica entre adolescentes que vivem com HIV/Aids por transmissão vertical. Este grupo encontra-se especialmente suscetivel por apresentar fatores de vulnerabilidade como doença crônica, orfandade com consequente troca de cuidadores e comprometimento da imagem corporal. A pesquisa foi realizada com adolescentes atendidos em um hospital de referência. A primeira etapa abrangeu a aplicação dos instrumentos Parent Child Conflict Tactics Scales e Escala de Violência Psicológica, para aferição do tipo de violência intrafamiliar acometida. A segunda parte, qualitativa, utilizou-se de entrevistas com os adolescentes, com os maiores e os menores escores para violência nos instrumentos quantitativos. Verificou-se uma alta prevalência de violência física e psicológica, bem como nas entrevistas realizadas foram detalhadas as dinâmicas familiares abusivas, corroborando a literatura. Tendo em vista as consequências deste tipo de violência, se torna fundamental que o profissional de saúde esteja atento à identificação das situações de violência, como também ao reconhecimento dos fatores de vulnerabilidade e ao incentivo aos fatores de proteção aos maus-tratos.

Palavras-chave Violência intrafamiliar; HIV/ Aids, Fatores de vulnerabilidade, Fatores de proteção 


\section{Introdução}

A violência intrafamiliar contra crianças e adolescentes é um fenômeno complexo e multicausal, onde formas agressivas e cruéis de tratamento podem ser usadas pelos pais ou por algum outro membro da família, incluindo indivíduos que passam a assumir função parental ${ }^{1,2}$.

O modelo ecológico preconizado pela Organização Mundial de Saúde (OMS/WHO) reconhece a interação de fatores individuais da criança ou do adolescente, de relacionamento entre familiares ou amigos, dos que dizem respeito à comunidade e ao ambiente cultural, social e econômico como responsáveis pela maior suscetibilidade ou proteção à violência ${ }^{2-4}$. A análise destes aspectos permite o reconhecimento de fatores de vulnerabilidade e de proteção aos maus-tratos.

Vulnerabilidade é o conjunto de aspectos que vão além do nível individual, englobando aspectos coletivos, que levam a maior suscetibilidade aos agravos, e a menor capacidade de se proteger $^{5}$. O conceito de vulnerabilidade surgiu no cenário da saúde pública no início da década de 80 , a partir do aparecimento da Aids, numa tentativa de explicar a tendência individualizante da doença. É diferente do conceito de risco, utilizado em estudos epidemiológicos, que significa a probabilidade que um indivíduo tem em apresentar determinado desfecho quando exposto a uma ou mais variáveis, deixando de pertencer a um determinado grupo identitário (expostos), para pertencer a outro grupo (afetados) ${ }^{6,7}$.

Dentre os fatores de vulnerabilidade à violência familiar apontados pela literatura destacamse: condições de pobreza; falta ou precariedade de moradia; famílias em situação de crise (pais desempregados, separação do casal, doença crônica e/ou incapacitante, morte); famílias baseadas numa distribuição desigual de autoridade e poder ou cujas relações são centradas em papéis rigidamente definidos; família monoparental; pais ou cuidadores que consideram a punição física como método de disciplina ou que demonstram falta de controle ao serem contrariados ou diante de circunstâncias de tensão; história de violência intrafamiliar; prole numerosa; ausência de uma rede de apoio em situações de dificuldade; baixa autoestima; isolamento social; dependência química; antecedentes criminais ${ }^{2,3,8-10}$.

Já os fatores de proteção são divididos em três tipos: individuais (autoestima positiva, autocontrole, autonomia e sonhos pessoais definidos para o futuro), familiares (relacionamentos familiares pautados no apoio mútuo e proteção entre membros, diálogo aberto, preservação da individualidade do filho, presença de rede de apoio, identificação de figuras positivas na família e desenvolvimento de habilidades para resolução não violenta de conflitos) e sociais (participação em programas de auxílio e em projetos para o reconhecimento precoce de sinais e sintomas de violência e atividades que aumentem a autoestima e melhorem a autoimagem corporal dos adolescentes) $)^{2,11-15}$.

Adolescentes que vivem com HIV/Aids por transmissão vertical, formam um grupo singular de análise. Além de serem portadores de doença crônica e estigmatizante, o que pode levar a maior vulnerabilidade e à violência intrafamiliar, também podem apresentar déficits quanto ao crescimento e desenvolvimento, sequelas por doenças anteriores, vários episódios de internação e diversos efeitos colaterais pelo longo uso dos medicamentos antiretrovirais. Assim sendo, por se sentirem diferentes em relação aos seus pares, os adolescentes podem apresentar baixa autoestima e isolamento do grupo, aumentando ainda mais a suscetibilidade à violência intrafamiliar ${ }^{8,16,17}$. A Aids afeta as famílias como um todo, principalmente no caso da transmissão vertical, onde muitos pais encontram-se doentes ou falecidos. A orfandade e a troca frequente de cuidadores são vivências comuns a este grupo. Todos esses fatores descritos podem gerar sofrimento, tornando esses adolescentes muitas vezes, ainda mais vulneráveis aos maus-tratos ${ }^{16,18,19}$.

Este artigo se propôs a analisar os fatores de vulnerabilidade e de proteção à violência intrafamiliar física e psicológica contra adolescentes que vivem com HIV/Aids, infectados por transmissão vertical.

\section{Metodologia}

Foi realizado um estudo exploratório, do tipo quantitativo e qualitativo, entre os meses de maio a novembro de 2008.

O local escolhido foi um ambulatório de doenças infecciosas pediátricas no Rio de Janeiro, por ser um importante polo de saúde deste estado, sendo referência no atendimento de crianças e adolescentes que vivem com HIV/Aids.

Foram sujeitos do estudo 31 dos 34 adolescentes entre 12 e 18 anos, infectados por transmissão vertical (mãe-filho) que estavam em acompanhamento ambulatorial. Três adolescentes não participaram do estudo: um por recusa e dois por evasão às consultas. 
Embora o número de adolescentes que participaram deste estudo possa parecer pouco expressivo, deve-se ressaltar que o grupo estudado é composto exclusivamente por adolescentes que foram contaminados por transmissão vertical. Essa forma de transmissão não é a mais comum, visto que a maioria dos adolescentes adquire a infecção por via sexual ${ }^{16,20}$. O grupo estudado sobreviveu graças aos avanços obtidos no manejo terapêutico da doença, que os permitiu alcançar esta faixa etária.

\section{Estudo Quantitativo}

Foram aplicados dois instrumentos mensuradores de violência física e psicológica, o questionário Parent-Child Conflict Tactics Scales $(\mathrm{CTSPC})^{21}$ e a Escala de Violência Psicológica ${ }^{22}$, adaptados transculturalmente no Brasil por Reichenheim e Moraes ${ }^{23}$ e por Avancini et al. ${ }^{22}$, respectivamente. Estes instrumentos foram aplicados em todos os adolescentes que vivem com HIV/Aids atendidos num ambulatório de Doenças Infecciosas Pediátricas que concordaram em participar da pesquisa.

Um profissional treinado, que desconhecia a história pregressa dos adolescentes, aplicou os questionários em forma de entrevista, já que alguns deles apresentavam dificuldade na leitura do texto. Os dois instrumentos foram aplicados no mesmo dia. $\mathrm{O}$ adolescente respondeu às perguntas dos questionários sem a presença dos seus pais ou cuidadores, em um espaço previamente estabelecido, sem determinação de tempo para resposta.

O instrumento CTSPC tem como objetivo mensurar maus-tratos físicos e psicológicos na relação pais-crianças ou adolescentes, bem como os modelos não violentos de disciplina ${ }^{24}$, classificando as respostas como: disciplina não violenta, agressão psicológica e maus-tratos físicos. Os maus-tratos físicos são divididos em três grupos de acordo com a gravidade da agressão: punição corporal, maus-tratos físicos graves, e maus-tratos físicos gravíssimos ${ }^{21,24,25}$. A pontuação é calculada dependendo do objetivo da aplicação do instrumento. Como nesta pesquisa a finalidade era calcular a prevalência de violência, o escore 1 indicava um ou mais atos de violência sofrido no último ano (ou em algum momento anterior ao último ano), enquanto o score 0 (zero) significava ausência de ato violento. Os itens relacionados à agressão física diferem muito em relação à gravidade, e a escala foi desenvolvida para levar em conta essa importante diferença. Assim sen- do, a pontuação dos diferentes tipos de agressão física variou de acordo com a seriedade do ato, onde os mais graves tiveram escores mais altos do que os menos graves ${ }^{25,26}$.

A Escala de Violência Psicológica se propõe a avaliar experiências negativas de crianças e adolescentes em algum momento de sua infância ou adolescência, proporcionadas por alguém importante em suas vidas, abrangendo aspectos de humilhação, culpa, crítica, desqualificação, desencorajamento, agressão verbal, recusa de afeto, responsabilização excessiva e falta de interesse. Cada resposta variou de um a cinco pontos, e o maior escore indicou presença de violência psicológica severa ${ }^{22}$. Embora tenha sido utilizada a contagem de pontos como estratégia de seleção para a etapa qualitativa, foram considerados como vítima de violência intrafamiliar os adolescentes que responderam "quase sempre" e/ou "sempre" em alguma das respostas da escala. Por este instrumento ser mais detalhado em relação à violência psicológica, quando comparado ao CTSPC, resolveu-se utilizar apenas o resultado desse questionário como critério de identificação de maus-tratos psicológicos.

A utilização destes instrumentos serviu como estratégia metodológica para identificar dentre estes adolescentes, quais sofriam violência intrafamiliar e de que tipo, física e/ou psicológica, eram vítimas.

\section{Estudo Qualitativo}

A segunda etapa constou de entrevistas semiestruturadas com os adolescentes que responderam previamente os questionários, e apresentaram os maiores escores por categoria. Como não ocorreu nenhum caso na categoria maus-tratos físicos graves, optou-se por entrevistar o adolescente que apresentou os maiores escores em todas as categorias de violência física e psicológica. Portanto, foram entrevistados quatro adolescentes na categoria disciplina não violenta e quatro na categoria violência.

A análise das entrevistas foi realizada segundo os procedimentos da análise temática: leitura compreensiva do material selecionado, categorização (dividindo o material em partes, e estas partes em categorias, descrevendo-as), inferência (dedução dos resultados), descrição e interpretação (pautado no marco teórico adotado) dos resultados encontrados ${ }^{27}$.

Os principais temas abordados neste artigo e suas respectivas categorias empíricas nucleadoras de sentido foram: ambiente familiar ("ambi- 
ente em paz" x "local difícil de viver"/"muito estressado”); relação do adolescente com os seus responsáveis ("conversa e respeito entre si” $\mathrm{x}$ "não conversa, mas discute”); conflitos familiares envolvendo os adolescentes ("pode bater/não pode bater", "implicância entre irmãos", "não gosto de fazer estas coisas", "sempre tem alguma coisa para reclamar”). Nesta etapa da pesquisa também foi perguntado sobre as situações de violência e de proteção identificadas no questionário respondido na primeira fase do trabalho.

Para a análise final dos resultados, foi realizada a triangulação entre os dados obtidos através da aplicação dos instrumentos CTSPC, Escala de Violência Psicológica e da análise das entrevistas.

\section{Resultados e Discussão}

\section{Convivendo sem Violência}

Os adolescentes que apresentaram os maiores escores para disciplina não violenta foram entrevistados e a análise destas entrevistas mostrou que nestas famílias a convivência era caracterizada pela definição clara dos papéis, pela presença do diálogo, pela negociação dos conflitos e pelo respeito entre seus membros. A negociação diante de conflitos entre cuidadores e adolescentes eram bem sucedidas, agradando a todos. Uma categoria empírica que ilustra este grupo é definida pela expressão "sentar prá conversar" (A gente senta prá conversar, a gente tenta sempre conversar $[\mathrm{A} 14])$.

Percebe-se que neste grupo de adolescentes sem violência familiar, a presença dos fatores de proteção foi bastante significativa, encontrando-se principalmente dentre os fatores individuais, o exercício da autonomia que apareceu de diversas formas nos relatos. Se por um lado, um adolescente recebia mesada e podia gerenciá-la do jeito que achasse melhor, o outro podia sair com os amigos para vários lugares sem estar acompanhado da mãe, como, de um modo geral, acontece com os adolescentes que não vivem com HIV/ Aids.

A educação destes adolescentes se dava através de um diálogo aberto, respeito entre seus membros e preservação da individualidade do filho. Este achado é corroborado por estudos com adolescentes no Brasil, México e Espanha ${ }^{10,15,28}$.

Em nosso estudo, foi perguntado aos adolescentes o que eles consideravam importante para o bom relacionamento em casa, resgatando assim, as respostas que tinham sido dadas por eles no momento da aplicação dos questionários. O respeito entre os membros da família e o diálogo foram mais uma vez citados, capazes de transformar o "ambiente em paz":

O respeito, sentar prá conversar, a paz assim, são três itens que a gente tem lá em casa, sempre respeitando um ao outro, se não respeitar, vira uma bagunça! Filho prá um lado, mãe pro outro, acho que isso sim, transforma o ambiente em paz. (A6)

\section{Convivendo com Violência Física}

A análise da aplicação do instrumento $\mathrm{Pa}$ rent-Child Conflict Tactics Scales mostrou que os maus-tratos contra adolescentes tiveram significativa prevalência, corroborando com estudos realizados pela OMS/WHO ${ }^{2}$. Dentre os adolescentes entrevistados, $42 \%$ sofreu punição corporal no último ano, sendo que destes, cinco (16\%) já tinham sido vítimas anteriormente. $\mathrm{O}$ tipo de agressão mais encontrado foi "tapa na mão, no braço ou na perna”. (Tabela 1)

Em relação aos maus-tratos físicos graves, verificou-se que $22,6 \%$ dos adolescentes tinham sido vítimas deste tipo de violência física no último ano. A forma de violência empregada foi unânime, isto é, "bater em alguma parte do corpo diferente do bumbum, com alguma coisa como um chinelo, escova de cabelo, vara ou outro objeto duro".

Não houve relato de caso na categoria maustratos físicos gravíssimos.

Ficou evidente ao analisar os questionários que a maior responsável pela violência infringida à criança e ao adolescente foi a mãe, conforme mostra a literatura ${ }^{9,29}$. Segundo estes autores, em mais de $80 \%$ dos casos a mãe foi apontada como

Tabela 1. Tipos de violência intrafamiliar física encontrados na aplicação dos questionários.

\begin{tabular}{lrr}
\hline & \multicolumn{2}{c}{$\mathbf{n}=\mathbf{3 1}$ adolescentes } \\
\cline { 2 - 3 } \multicolumn{1}{c}{ Tipo de violência } & $\mathbf{n}$ & $\%$ \\
\hline Punição corporal & & \\
$\quad$ passado & 15 & 48,3 \\
$\quad$ último ano & 13 & 42 \\
$\quad$ último ano/passado & 5 & 16,1 \\
Maus-tratos físicos graves & & \\
$\quad$ último ano & 7 & 22,6 \\
passado & 3 & 9,7 \\
$\quad$ último ano/passado & 0 & 0 \\
\hline
\end{tabular}


quem mais utilizou de violência para com os filhos. Embora o presente estudo também tenha mostrado a mãe como o agente agressor mais comum, o resultado percentual encontrado (35\%) foi menor em relação à literatura. Como o adolescente que vive com HIV/Aids por transmissão vertical muitas vezes tem pais falecidos, a presença do cuidador como causador da violência familiar (20\%) se apresentou de maneira mais expressiva. Também tiveram relevância os seguintes agentes agressores: mãe e cuidador $(22,5 \%)$; pai e cuidador $(9,6 \%)$; pai e mãe $(6,45 \%)$.

Segundo Gonçalvo ${ }^{9}$ e Werber et al. ${ }^{29}$, os motivos que levariam as mães a agredirem seus filhos seriam: responsabilidade materna na educação dos filhos; a maior presença materna em casa, por ser grande o número de mulheres que não trabalham fora; a existência de famílias monoparentais maternas.

As entrevistas destes adolescentes mostraram um ambiente doméstico definido como um "local difícil de viver", sintetizado pela categoria "muito estressado". O enfrentamento das situações de conflito por parte das famílias era baseado na agressão física, conforme o relato:

A sensação que me dá é que por qualquer coisinha ela (a mãe) quer me bater! (A3)

É muito estressado lá. (A20)

Dentre as causas que geram os conflitos, encontraram-se a recusa à realização de algumas tarefas domésticas solicitadas pelos cuidadores, especialmente para as meninas e a rivalidade entre irmãos como causadores de agressões física. Estes conflitos são, de maneira geral, muito comuns nesta faixa etária. A pesquisa realizada por Assis e Constantino ${ }^{30} \mathrm{com}$ adolescentes privadas de liberdade mostrou que as causas mais comuns de conflito familiar são: desrespeito às normas familiares, recusa em ajudar nos serviços domésticos, namoros, falta à escola, uso de cigarros e drogas e ciúmes do padrasto.

A fala dos entrevistados argumenta o ponto de vista deste grupo e de sua recusa a ajudar nas tarefas ("eu não consigo gostar de fazer essas coisas") e ilustram as competições travadas entre irmãos, denominadas por eles como "implicância".

Ele fica assim implicando... É que ele não quer lavar a louça, e começa a falar, a implicar assim comigo... [...] aí eu falo que 'vou falar com a minha mãe, e ele me bate. Conto (para a mãe). E aí ela bate nele. (A3)

A família de A3 vivia sob constante violência, envolvendo todos os seus membros. A mãe abusava fisicamente não só da filha, como também de outros familiares, inclusive do filho mais velho, que por sua vez, agredia a menina física e psicologicamente. Somente o pai de A3 não a maltratava. Este tipo de situação é retratada por Straus e Gelles ${ }^{31}$, que realizaram um estudo sobre violência nos Estados Unidos onde entrevistaram 8.145 famílias e verificaram que, naquelas onde a mulher agredia fisicamente o marido, como também era o caso de A3, a criança tinha 2,2 mais chances de ser agredida por sua mãe, quando comparado com famílias sem violência entre os casais. Segundo Hornor ${ }^{32}$, aproximadamente $40 \%$ das crianças que testemunham a violência intrafamiliar também são agredidas fisicamente.

Reichenheim et al. ${ }^{33}$ afirmam que crianças que são vítimas de violência intrafamiliar ou que testemunham a violência entre os pais podem, quando adultos apresentar distúrbios emocionais, cognitivos e comportamentais. Estudos revelam que essas crianças e adolescentes podem se tornar futuros perpetradores ou futuras vítimas de violência, incluindo suicídio, abuso sexual, violência entre jovens e entre casais ${ }^{2,34}$. A3 e seu irmão vivenciaram essa situação, e ele, agora adulto, repete com ela o modelo de comportamento violento ao qual foi exposto. Weber et al. ${ }^{29}$ afirmam que esta passagem de modelo de geração para geração é um dos motivos que fazem com que a punição corporal como prática educativa seja mantida ao longo dos séculos.

A punição física como medida educativa foi mencionada por alguns adolescentes entrevistados. Frequentemente os cuidadores confundem punição corporal com disciplina, utilizando a força física para a correção ou mudança de comportamento das crianças e dos adolescentes ${ }^{2,34}$. Disciplinar, por outro lado, seria o uso de estratégias positivas reforçando a autoestima e a habilidade de saber se comportar apropriadamente, construindo relacionamentos positivos ${ }^{29}$.

Para a criança ou adolescente, o pai ou o cuidador detêm o poder hierárquico, tendo autoridade para dar ordens, tomar decisões em relação a eles, e consequentemente, dispor do legítimo direito de bater para assim educar, em nome do próprio bem deles. A20, que mora com o pai, dois irmãos, a madrasta e o filho dela, exemplificou essa situação, expressada pelas categorias "pode bater" e "não pode bater":

“Às vezes o $M$. (irmão de consideração) fica respondendo ele, ele (o pai) dá nervos que bate, mas não pode bater. Porque meu pai não é o pai dele, não é? Pode (bater nela e nos irmãos). Porque ele é meu pai. (A20) 


\section{Convivendo com Violência Psicológica}

A Escala de Violência Psicológica possibilitou a identificação deste tipo de violência em vinte $e$ um $(67,7 \%)$ destes adolescentes, superando os dados apontados pela literatura, para adolescentes sem o status sorológico conhecido. Estudo realizado por Assis et al. ${ }^{35}$ com 1.460 adolescentes de escolas públicas e particulares, utilizando como referência esta escala e o instrumento CTS mostrou que havia predominância do abuso psicológico, atingindo $48 \%$ dos estudantes investigados.

Um outro estudo realizado no Peru por Sullica e Schirmer ${ }^{36}$, com 472 adolescentes grávidas, indicou que em 99,5\% dos casos onde se constatou a violência física, esta foi precedida da violência psicológica. Silva et al. ${ }^{37}$ reafirmam essa questão, colocando que a violência psicológica é o ponto inicial que, de uma maneira geral, deflagra os outros tipos de violência doméstica.

No presente estudo, os diferentes tipos de violência se mesclaram, e nos resultados verificou-se que os adolescentes que apresentavam os maiores escores em violência psicológica eram os mesmos que apresentavam os maiores escores para punição corporal e maus-tratos físicos moderados. A exceção foi A19, que apresentou a maior pontuação para violência psicológica, e uma das menores para qualquer tipo de agressão física.

Dentre as diversas formas de violência psicológica, este trabalho identificou pelo menos um relato de cada tipo de agressão psicológica apresentada na Escala de Violência Psicológica. Straus e Field ${ }^{25}$ realizaram uma pesquisa com 991 pais, que mostrou que independentemente das características sociodemográficas, quase todos eles usaram como tática disciplinar, pelo menos um tipo de agressão psicológica descrita no questionário CTSPC.

A crítica excessiva, uma das formas de violência psicológica, foi apontada por adolescentes e sintetizada pela categoria "sempre tem alguma coisa para reclamar":

Não sei por que é assim. Sempre que eu faço alguma coisa, a minha vó sempre tem alguma coisa prá reclamar, e eu pego e não faço mais [...]. Não sei como explicar, é um negócio estranho. (A10)

$A h$, às vezes, quando faço besteira, eles (os avós) falam, só que às vezes, não tem motivo de brigar comigo, e eles brigam... (A19)

As relações diante dos conflitos familiares é pautada pelo não diálogo e por muitas agressões verbais. As falas apontam a categoria "não conversa, mas discute” como a expressão empírica desta prática familiar:
Ah, ela (a avó) é muito estranha, a gente começa a discutir, aí ela para de falar comigo [...] (A10)

Aos adolescentes que eram vítimas de violência intrafamiliar foi perguntado, ao final de cada entrevista o que gostariam de mudar nas relações familiares, para que a convivência entre seus membros fosse mais harmônica e feliz. Pode ser observado que nenhum dos adolescentes vítimas de violência referiu à mudança de comportamento do seu cuidador, que era o seu agressor, como medida para a melhoria das relações familiares. Ao invés disso, isentaram os cuidadores da responsabilidade pelas agressões.

[...] eu não consigo ficar longe da minha mãe. Até quando eu vou nas férias na casa da minha avó, eu já fico com saudades [...] A minha mãe tem os defeitos dela, mas ela é legal. Tudo que eu peço a ela, tudo o que ela pode me dar, ela me dá. Ela me manda estudar, diz que é para o meu bem. Que eu não estou estudando prá ela, e sim prá mim mesmo, prá não depender de homem [...]. (A3)

Esta questão é apontada por Assis e Constantino $^{30}$ em um trabalho sobre a infração juvenil feminina. Embora essas jovens fossem submetidas a todo tipo de sofrimento infringido por suas mães, elas amenizavam tais atos, por se sentirem culpadas pelo comportamento materno agressivo, como se fossem merecedoras das agressões.

\section{Conclusão}

Conforme observado neste estudo e atestado pela literatura, adolescentes que vivem com HIV/Aids por transmissão vertical apresentam alta prevalência de violência intrafamiliar, seja ela física ou psicológica

A aplicação de instrumentos considerados eficazes na detecção dos diferentes tipos de violência intrafamiliar e a realização de entrevistas possibilitaram não só a identificação de quais adolescentes se encontravam em situação de maus-tratos, como também foram eficazes no reconhecimento dos fatores de vulnerabilidade e de proteção.

A utilização dos instrumentos CTSPC e a Escala de Violência Psicológica na prática pelos profissionais de saúde que lidam com adolescentes que vivem com HIV/Aids pode servir como estratégia para a detecção destes casos.

As entrevistas realizadas na segunda etapa deste trabalho foram essenciais para mostrar como viviam e convivam os adolescentes, vítimas ou não de violência intrafamiliar, dando uma 


\section{Colaboradores}

ACMW Barros trabalhou na pesquisa bibliográfica, entrevistas, elaboração e redação do trabalho; OM Bastos na aplicação dos questionários, orientação metodológica, revisão do trabalho; MVS Pone na aplicação dos questionários, revisão crítica do trabalho; SR Deslandes na orientação metodológica, revisão final do trabalho famílias daqueles que não foram vítima de violência intrafamiliar, interferindo positivamente na proteção dos mesmos.

Deste modo, além da identificação dos fatores de vulnerabilidade aos maus-tratos conforme já assinalado, esforços multidisciplinares das equipes ligadas ao cuidado destes jovens devem ser feitos para reforçar os fatores de proteção, tais como relações familiares baseadas no diálogo e no respeito, rede de apoio e desenvolvimento de habilidades para a resolução não violenta de conflitos.

Tendo em vista que muitos dos fatores de vulnerabilidade encontrados neste grupo, e descritos na literatura, fogem do âmbito exclusivo da saúde, impõe-se a articulação intersetorial, no sentido de uma prevenção mais eficaz dos maustratos a crianças e adolescentes que têm doença crônica, e mais especificamente para os que vivem com HIV/Aids.

\section{Referências}

1. Brasil. Ministério da Saúde (MS). Notificação de maus-tratos contra crianças e adolescentes. Brasília: MS; 2002.

2. World Health Organization (WHO). International society for prevention of child abuse and neglect. Preventing child maltreatment: a guide to taking action and generating evidence. Geneva: WHO; 2006.

3. Krug EG, Dahberg LL, Mercy JA, Zwi AB, Lozano $\mathrm{R}$. World report on violence and health. Geneva: World Health Organization; 2002.

4. World Health Organization (WHO). Preventing violence: a guide to implementing the recommendations of the World Report on Violence and Health. Geneva: WHO; 2004.

5. Ayres JRCM, Calazans GJ, Saletti Filho HC. Adolescência e vulnerabilidade ao HIV/Aids: avaliação de uma estratégia de prevenção entre escolares de baixa renda na cidade de São Paulo. Divulg. saúde debate 2003; 29:93-114.

6. Callazans GJ, Saletti-Filho HC, Fança-Junior I, Ayres JRCM. O conceito de vulnerabilidade. In: Padoim SMM, organizador. Experiências interdisciplinares em Aids: interfaces de uma epidemia. Santa Maria: UFSM; 2006. p. 43-62.

7. Sánchez AIM, Bertolozzi MR. Pode o conceito de vulnerabilidade apoiar a construção do conhecimento em saúde coletiva? Cien Saude Colet 2007; 12(2):319-324. 
8. Brasil. Ministério da Saúde (MS). Secretaria de Políticas de Saúde. Violência intrafamiliar: orientações para práticas em serviço. Brasília: MS; 2001.

9. Gonzalvo GO. Maltrato en niños con discapacidades: características y factores de riesgo. Anales Españoles de Pediatria 2002; 56(3):219-223.

10. Suárez L, Menkes C. Violencia familiar ejercida en contra de los adolescentes mexicanos. Rev Saude Publica 2006; 40(4):611-619.

11. Brasil. Portaria MS/GM n. 737, de 16 de maio de 2001. Política nacional de redução da morbimortalidade por acidentes e violência. Diário oficial da União 2001; 18 de maio.

12. International Society for Prevention of Child Abuse and Neglect (IPSCAN). World Perspectives on Child Abuse. Chicago: IPSCAN; 2006.

13. Pesce RP, Assis SG, Santos N, Oliveira RVC. Risco e proteção: em busca de um equilíbrio promotor de resiliência. Psic. Teor. e Pesq. 2004; 20(2):135-143.

14. Assis SG, Deslandes SF, Santos NC. Violência na Adolescência sementes e frutos de uma sociedade desigual. In: Souza ER, Minayo MCS, organizadores. Impacto da violência na saúde dos brasileiros. Brasília: Ministério da Saúde; 2005. p. 80-115.

15. Gonzalvo GO. ¿Qué se puede hacer para prevenir la violencia y el maltrato de los niños con discapacidades? An Pediatr (Barc) 2005; 62(2):153-157.

16. Brasil. Ministério da Saúde (MS). Secretaria de Vigilância em Saúde. Programa Nacional de DST e Aids. Recomendações para terapia antirretroviral em crianças e adolescentes infectados pelo HIV. Brasília: MS; 2009.

17. Wierner LS, Battles HB, Wood LV. A Longitudinal Study of adolescents with perinatally or transfusion acquire HIV infection: sexual knowledge, risck reduction self-efficacy and sexual behavior. AIDS Behav 2007; 11(3):471-478.

18. França-Junior I, Doring M, Stella IM. Crianças órfãs e vulneráveis pelo HIV no Brasil: onde estamos e para onde vamos? Rev Saude Publica 2006; 40(Supl.): 23-30.

19. Nothomang K, Phaladze N, Oagile N, Ngwenya B, Seboni N, Gobotswang K. People living with HIV and Aids on the brink: stigma-a complex sociocultural impediment in the fight against HIV and Aids in Boswana. Health Care Women Int 2009; 30(3):233234.

20. UNAIDS. Aids epidemic update - special report on HIV/Aids. December 2010. Geneva: UNAIDS; 2010.

21. Straus MA, Hamby SL. Measuring physical and psychological maltreatment of children with the conflict tactics scales. In: Kaufman K, Jasinski G, Jana $\mathrm{L}$, organizadores. Out of the darkness: contemporary perspectives on family violence. Thousand Oaks, CA: Sage; 1997. p. 119-135.

22. Avanci JQ, Assis SG, Santos NC, Oliveira RVC. Escala de violência psicológica contra adolescentes. Rev Saude Publica 2005; 39(5):702-708.

23. Reichenheim ME, Moraes CL. Adaptação transcultural do instrumento Parent-Child Conflict Tactics Scales (CTSPC) utilizado para identificar a violência contra a criança. Cad Saude Publica 2003; 19(6): 1701-1712.
24. Straus MA, Hamby SL, Finkelhor D, Moore DW, Runyan D. Identification of child maltreatment with the Parent-Child Conflict Tactics Scales: development and psychometric data for a national sample of american parent. Child Abuse Negl 1998; 22(4):249-270.

25. Straus MA, Field CJ. Psychological aggression by American parents: national data on prevalence, chronicity, and severity. Journal of Marriage and Family 2003; 65(11):795-808.

26. Straus MA. Scoring the CTS2 and CTSPC. [acessado 2009 jan 5]. Disponível em: http://pubpages.unh. edu/ mas2/CTS28.pdf

27. Bauer MW. Análise de conteúdo clássica uma revisão. In: Bauer MW, Gaskell G, organizadores. Pesquisa qualitativa com texto, imagem e som. Petrópolis: Vozes; 2002. p.189-217.

28. Assis SG, Avanci JQ. Labirinto de espelhos: formação da auto-estima na infância e na adolescência. Rio de Janeiro: Fiocruz; 2004.

29. Weber LND, Viezzer AP, Branderburg OJ. O uso da palmada e surras como prática educativa. Estudos de Psicologia 2004; 9(2):227-237.

30. Assis SG, Constantino P. Filhas do Mundo: infração juvenil feminina no Rio de Janeiro. Rio de Janeiro: Fiocruz; 2001.

31. Straus MA, Gelles RJ. Physical violence in american families: risk factors and adaptations to violence in 8,145 families. New Jersey: Transaction Publishers; 1995.

32. Hornor G. Domestic violence and children. J Pediatr Health Care 2005; 19(4):206-212.

33. Reichenheim ME, Hasselmann MH, Moraes CL. Conseqüências da violência familiar na saúde da criança e do adolescente: contribuições para a elaboração de propostas de ação. Cien Saude Colet 1999; 4(1):109-121.

34. Straus MA. Physical Abuse. In: Straus MA, organizador. Beating the devil out of them. Corporal Punishment in American Families and its Effects on Children. New Jersey: Transaction Publishers; 2009. p. 81-97.

35. Assis SG, Avanci JQ, Santos NC, Malaquias JV, Oliveira RVC. Violência e representação social na adolescência no Brasil. Rev Panam Salud Pública 2004; 16(1):43-51.

36. Sullica TF, Schirmer J. Violência Intrafamiliar na adolescência na cidade de Puno-Peru. Rev Lat. Am. Enfermagem 2006; 14(4):578-585.

37. Silva LL, Coelho EBS, Caponi SNC. Violência silenciosa: violência psicológica como condição da violência física doméstica. Interface Comun Saúde Educ 2007; 11(21):93-103.

Artigo apresentado em 28/03/2012

Aprovado em 29/08/2012

Versão final apresentada em 20/09/2012 